\title{
Trial watch
}

\section{DETECTION OF DUCTAL CARCINOMAINSITU}

Previous studies have concluded that magnetic resonance imaging (MRI) is less sensitive than mammography for detecting the pre-invasive breast cancer, ductal carcinoma in situ (DCIS), but now a prospective observational study shows that MRI could help diagnose high-grade DCIS that is not detected by mammography.

7,319 women underwent both mammography and MRI for diagnostic assessment. All 1,208 women with a positive imaging diagnosis were biopsied to confirm mammographic microcalcifications, low-grade DCIS or high-grade DCIS. Of the 167 women who had both preoperative imaging tests and a final diagnosis of pure DCIS, $56 \%$ were diagnosed by mammography and $92 \%$ by MRI. MRI detected $98 \%$ of the high-grade DCIS cases, and $48 \%$ of these were missed by mammography.

Previous studies have investigated the diagnostic capability of MRI to detect DCIS in patients who had already had microcalcifications picked up by mammography, and have concluded that MRI is less sensitive than mammography. The results of the current study indicate that MRI detects a different subset of DCIS that do not show microcalcifications and are undetectable by mammography. The authors conclude that a systematic multi-institutional screening trial to investigate the role of MRI in diagnosing DCIS is required.

ORIGINAL RESEARCH PAPER Kuhl, C. K. et al. MRI for diagnosis of pure ductal carcinoma in situ: a prospective observational study. Lancet 370, 485-492 (2007)

\section{HAZARDS OF SEQUENTIAL KINASE INHIBITOR THERAPY}

$A B L$ kinase inhibitor therapy for chronic myeloid leukaemia $(C M L)$ is currently given sequentially: newly diagnosed patients receive imatinib, then the second-generation inhibitor dasatinib on relapse. Charles Sawyers and colleagues now warn that this approach can lead to compound drug-resistant BCR$A B L$ mutations, sometimes with increased oncogenic potency.

The authors analysed the BCR-ABL genotypes of 17 patients with CML who relapsed after sequential treatment with imatinib and dasatinib. While 12 patients relapsed with the pan-resistant T315I mutation, 6 patients had additional BCR$A B L$ mutations that conferred resistance to dasatinib in vitro but were predicted not to confer resistance to imatinib. Three of these patients were retreated with imatinib or with the imatinib-related compound nilotinib and responded. The compound mutants were shown to have increased oncogenic potency in transformation assays compared with each mutant alone. In addition, the Aurora kinase inhibitor, VX-680, which is active against the $T 315 \mathrm{I}$ mutation, is active against another common dasatinib-resistant mutation in their analysis, V299L.

A role for combining $A B L$ inhibitors, perhaps including VX-680, in the treatment of CML should be investigated further.

ORIGINAL RESEARCH PAPER Shah, N. P. et al. Sequential ABL kinase inhibitor therapy selects for compound drug-resistant BCR-ABL mutations with altered oncogenic potency.J. Clin. Invest. 16 August 2007 (doi:10.1172/JCI130,890) 